\title{
Isolation of a Novel Platelet-Derived Growth Factor- Responsive Precursor from the Embryonic Ventral Forebrain
}

\author{
Andrew Chojnacki and Samuel Weiss \\ Genes and Development Research Group, Department of Cell Biology and Anatomy, University of Calgary, Faculty of Medicine, Calgary, Alberta, \\ T2N 4N1 Canada
}

\begin{abstract}
Oligodendrocyte progenitor cells express platelet-derived growth factor (PDGF) receptor- $\alpha$ and, when expanded in PDGF only, have been shown to generate oligodendrocytes and astrocytes but never neurons. Recent evidence suggests that oligodendrocytes are generated by a common progenitor that also generates neurons but not astrocytes. We used the neurosphere culture system to isolate embryonic ventral forebrain, PDGF-responsive precursors (PRPs). We report that the medial ganglionic eminence is the source of PRP-generated neurospheres and that the progeny can differentiate into parvalbumin-positive interneurons, oligodendrocytes, and astrocytes. Thyroid hormone and bone morphogenetic protein-2 (BMP-2) promote the mutually exclusive differentiation of oligodendrocytes and neurons, respectively, whereas ciliary neurotrophic factor acts with BMP-2 to suppress OLIG2 expression and promote astroglial differentiation. PRPs require fibroblast growth factor-2 together with PDGF to maintain self-renewal, which is dependent on sonic hedgehog signaling. We present evidence for forebrain oligodendrocytes and parvalbumin-positive interneurons being generated by a common precursor and elucidate signals regulating the multiple differentiation routes of the progeny of this precursor.
\end{abstract}

Key words: oligodendrocyte; precursor; PDGF; forebrain; Olig; BMP

\section{Introduction}

Although there is general agreement about the factors involved in the development of oligodendrocyte progenitors (OLPs) throughout the CNS, their precise phenotype potential is highly contentious (Liu and Rao, 2003; Noble et al., 2004; Rowitch, 2004). Findings in the spinal cord, which show that similar levels of sonic hedgehog $(\mathrm{SHH})$ signaling can induce motor neuron and oligodendrocyte cell fates (Pringle et al., 1996; Orentas et al., 1999) and that the basic helix-loop-helix transcriptional repressor Olig2 is required for the generation of both cell types (Lu et al., 2002), are consistent with the idea that oligodendrocytes and motor neurons are generated by a common progenitor. In the brain, crosses of Olig1-CRE and Rosa-lox- $\beta$-Gal mice have revealed mutually exclusive expression of $\beta$-galactosidase $(\beta$-Gal) and the astrocyte antigen $\mathrm{S} 100 \beta$, whereas neurons and oligodendrocytes were labeled with $\beta$-Gal (Lu et al., 2002), suggesting the latter were generated by a common precursor. However, the broad expression of OLIG1/2 in the embryonic forebrain compared with platelet-derived growth factor receptor- $\alpha$ (PDGFR $\alpha$ ) (Tekki-Kessaris et al., 2001), an early OLP antigen, makes it difficult to determine whether neurons are generated by embryonic OLPs or nonrelated OLIG-expressing progenitors. Additional support for a forebrain neuron/OLP comes from observations

\footnotetext{
Received Aug. 11, 2004; revised 0ct. 15, 2004; accepted 0ct. 20, 2004.

This work was supported by the Multiple Sclerosis Research Foundation of Canada and the Canadian Institutes of Health Research. S.W. is an Alberta Heritage Foundation for Medical Research Scientist. We are very grateful to the Genetics Institute for supplying BMP-2. We thank C. Schuurmans and C. Gregg for critical reading of a previous version of this manuscript and Dorothea Livingstone for excellent technical assistance.

Correspondence should be addressed to Dr. Weiss at the above address. E-mail: weiss@ucalgary.ca.

DOI:10.1523/JNEUROSCI.3302-04.2004

Copyright $\odot 2004$ Society for Neuroscience $\quad 0270-6474 / 04 / 2410888-12 \$ 15.00 / 0$
}

that the tangential migration of both GABAergic interneurons and oligodendrocytes is disrupted in $D l \times 1 / 2$ null mice (Yung et al., 2002). However, the fact that taulacZ-positive astrocytes appear in mice that express taulac $Z$ under the $D l \times 2$ promoter (Marshall and Goldman, 2002) suggests that DLX-expressing cells may be either multipotent or a heterogeneous population.

In contrast to the studies cited above, crosses of Olig1-CRE and Rosa-lox- $\beta$-Gal mice have also shown that some OLIG1expressing cells in the spinal cord eventually become astrocytes (Liu and Rao, 2004), thereby providing in vivo evidence for an oligodendrocyte/astrocyte progenitor. Furthermore, the in vitro isolation of glial-restricted precursors (GRPs) from the spinal cord (Rao and Mayer-Proschel, 1997; Rao et al., 1998), and their transplantation and differentiation into astrocytes and oligodendrocytes (Rao and Mayer-Proschel, 1997; Rao et al., 1998; Herrera et al., 2001), supports such a lineage model. However, the fact that GRPs can be isolated from dorsal as well as ventral embryonic spinal cords contrasts with studies demonstrating the ventral restriction of OLPs (Warf et al., 1991; Pringle and Richardson, 1993; Ono et al., 1995; Lu et al., 2002; Zhou and Anderson, 2002). This may be reconciled by the findings of Gabay et al. (2003), who found that the deregulation of dorsoventral patterning in vitro, attributable in part to aberrant $\mathrm{SHH}$ production induced by fibroblast growth factor (FGF) signaling, may be responsible for the generation of oligodendrocytes by dorsally derived GRPs. Nevertheless, a variety of studies in the brain, including in vivo retroviral-mediated lineage studies of the rat postnatal cerebral cortex (Levison et al., 1993; Levison and Goldman, 1993) as well as in vitro characterization of cortical OLPs (Mabie et al., 1997) and optic nerve oligodendrocyte type-2 astrocyte (O-2A) progenitors (Temple and Raff, 1985), which never generate neu- 
rons unless they are reprogrammed to become neural stem cells (NSCs) by their differentiation into astrocytes and subsequent expansion in FGF-2 (Kondo and Raff, 2000), supports the contention that astrocytes and oligodendrocytes are generated by a common progenitor. However, retroviral tracing of the prenatal rat cortex revealed that glial clones were either oligodendroglial or astroglial (Parnavelas, 1999), although the same study also found mixed oligodendrocyte and astrocyte clones when injections of retrovirus were made into the postnatal subventricular zone (SVZ). Interestingly, progenitors that express NG2, a chondroitin proteoglycan previously shown to colocalize to O-2A progenitors in vivo (Nishiyama et al., 1996), have been found to generate neurons in the postnatal hippocampus (Belachew et al., 2003). However, these progenitors express the epidermal growth factor receptor (EGFR) and have been identified as transitamplifying type C-like mulitpotent cells (Aguirre et al., 2004); therefore, whether they represent the differentiation properties of an OLP population is questionable.

Thus, exactly which cell types OLPs produce in the developing embryonic forebrain remains unclear. PDGFR $\alpha$ is one of the best-studied and earliest markers for OLPs in the CNS, including the forebrain. Surprisingly, we found that no studies have directly examined the phenotype potential of embryonic ventral forebrain, PDGF-responsive precursors (PRPs). Therefore, using PDGF as a mitogen for oligodendrocyte precursors, we examined the potential of embryonic day 14 (E14) ventral forebrain PRPs to generate neurons, oligodendrocytes, or astrocytes and to selfrenew. We used the neurosphere assay we developed (Reynolds et al., 1992) to study PRPs, to limit manipulation of the primary cells, and thereby to limit the effect of the in vitro environment. We found that clonally derived, neurosphere-generating PRPs are distinct from those generated by EGF-responsive NSCs. Specific differentiation signals reveal that ventral forebrain PRPs have the potential to generate neurons, oligodendrocytes, and astrocytes. We also found that PRPs have a limited capacity for self-renewal when passaged into PDGF, but this can be significantly enhanced by FGF-2, and that this cooperative process is dependent on SHH signaling. These findings suggest that selfrenewing PRPs may generate neurons and both types of macroglia during forebrain development.

\section{Materials and Methods}

Animals. Ubiquitous green fluorescent protein (GFP)-expressing [TgN(GFPU)5Nagy] mice were obtained from The Jackson Laboratory (Bar Harbor, ME) and along with CD-1 mice stocks were maintained in the University of Calgary Bioscience Animal Resources Center.

Cell culture. The culture medium [media hormone mix (MHM)] was composed of DMEM/F-12 (1:1) including 5 mM HEPES buffer, $0.6 \%$ glucose, $3 \mathrm{~mm}$ sodium bicarbonate, $2 \mathrm{~mm}$ glutamine, $25 \mu \mathrm{g} / \mathrm{ml}$ insulin, $100 \mu \mathrm{g} / \mathrm{ml}$ transferrin, $20 \mathrm{~nm}$ progesterone, $10 \mu \mathrm{M}$ putrescine, and $30 \mathrm{~nm}$ sodium selenite [all were from Sigma (St. Louis, MO), except glutamine was from Invitrogen (Carlsbad, CA)]. The lateral ganglionic eminence (LGE), medial ganglionic eminence (MGE), or both ganglionic eminences were removed from E14 mouse embryos and mechanically dissociated with a fire-polished Pasteur pipette in MHM. Cells were plated at a density of $0.01 \times 10^{6} \mathrm{cells} / \mathrm{ml}$, unless indicated otherwise. For the generation of neurospheres, PDGF-AA (100 ng/ml; Peprotech, Rocky Hill, NJ), PDGF-BB (100 ng/ml; Peprotech), EGF (20 ng/ml; Peprotech), FGF-2 (20 ng/ml; R \& D Systems, Minneapolis, MN) plus heparan sulfate (HS) $(2 \mu \mathrm{g} / \mathrm{ml}$; R \& D Systems), SHH ( $2 \mu \mathrm{g} / \mathrm{ml}$; R \& D Systems $)$, cyclopamine ( $5 \mu \mathrm{M}$; Toronto Research Chemicals, North York, Ontario, Canada), and/or DMSO (0.1\%; carrier) was added to the MHM. MHM used to generate neurospheres also contained 2\% B27 (Invitrogen). Neurospheres were differentiated on poly-L-ornithine-coated coverslips in MHM and in the presence or absence of 1\% FBS (Invitrogen), BMP-2
(Genetics Institute, Cambridge, MA), triiodothyronine (T3) (Sigma), and/or CNTF [generated as described previously (Gupta et al., 1992)] for 2-3 d in vitro (DIV). To determine whether PDGF-induced neurosphere generation was the result of clonal expansion, dissociated E14 MGEs from GFP and CD-1 albino mice were cultured in PDGF-AA (1:1) at a density of $0.02 \times 10^{6}$ cells $/ \mathrm{ml}$ for 6 DIV. The number of GFP-, nonGFP-, and chimeric GFP-expressing neurospheres was counted after 6 DIV using a Microsystems DMIL inverted fluorescence microscope (Leica, Richmond Hill, Ontario, Canada).

Self-renewal capacity was examined by single-sphere dissociation. Briefly, single 6 DIV neurospheres of equivalent size that were generated in the presence of EGF, PDGF-AA, or PDGF-AA plus SHH were transferred into 96-well plates and mechanically dissociated. Dissociates were cultured in MHM supplemented with EGF, FGF-2 (includes $2 \mu \mathrm{g} / \mathrm{ml}$ HS), FGF-2 plus SHH, PDGF-AA plus SHH, PDGF-AA plus FGF-2, PDGF-AA plus FGF-2 plus SHH, PDGF-AA plus DMSO (0.1\%), or PDGF-AA plus FGF-2 plus cyclopamine. The number of secondary neurospheres generated was counted after 9 DIV.

To test whether extrinsic factors could promote the self-renewal of PRPs, individual, 7 DIV, GFP-expressing, PDGF-generated neurospheres were isolated, dissociated in the presence of PDGF-AA, and differentiated on coverslips that had been plated $2 \mathrm{~d}$ earlier with or without $0.2 \times 10^{6}$ cells $/ \mathrm{ml}$ of EGF-generated cells from dissociated primary EGF neurospheres. EGF-generated cells had been allowed to differentiate for $2 \mathrm{~d}$ in the presence of $1 \%$ FBS. Plates were rinsed three times with MHM before the addition of GFP-expressing, PDGF-generated dissociates. The number of adherent clones and cells per clone was assessed by GFP expression. All images were captured with a Coolsnap digital camera (Photometrics, Tuscon, AZ) mounted on a Leica Microsystems DMIL inverted fluorescence microscope with Coolsnap version 1.2.0 software.

Immunofluorescence. Six DIV primary PDGF-AA-generated neurospheres were differentiated on poly-L-ornithine-coated coverslips and after 2 DIV were fixed for $20 \mathrm{~min}$ in $4 \%$ paraformaldehyde. For mouse IgM anti-O4 (1:10; Chemicon, Temecula, CA), coverslips were incubated in PBS, $\mathrm{pH} 7.5$, overnight at $4^{\circ} \mathrm{C}$. Coverslips were also incubated with mouse anti- $\beta$-III-tubulin (1:1000; Sigma), rabbit anti-GFAP (1:300; BTI, Stoughton, MA), mouse anti-GFAP (1:500; Chemicon), goat antimouse PDGFR $\alpha$ (1:10; R \& D Systems), rabbit anti-GFP (1:100; Santa Cruz Biotechnology, Santa Cruz, CA), rabbit anti-human myelin basic protein (MBP) (1:200; Dako, Mississauga, Ontario, Canada), mouse anti-neurofilament M (NFM) (1:50; RMO270; a gift from Dr. Virginia Lee, University of Pennsylvania, Philadelphia, PA), and/or rabbit antiOLIG2 (1:250; a gift from Dr. Masato Nakafuku, Division of Developmental Biology, Cincinnati Children's Hospital Research Foundation, Cincinnati, OH) in $0.3 \%$ Triton X-100 in PBS for $2 \mathrm{hr}$ at $37^{\circ} \mathrm{C}$. After incubation with primary antibodies, all tissue was incubated for $1 \mathrm{hr}$ in PBS and 10\% normal serum of the secondary antibody host [all secondary antibodies and reagents were from Jackson ImmunoResearch (West Grove, PA), except horseradish peroxidase (HRP)-conjugated secondary antibody was from Chemicon]. This was followed by a $1 \mathrm{hr}$ incubation with a biotin-conjugated secondary antibody and afterward a $1 \mathrm{hr}$ incubation at $37^{\circ} \mathrm{C}$ with streptavadin-cyanine 3 (Cy3) (1:1000) or streptavadin-FITC (1:500) for O4 staining. For the other primary antibodies, the coverslips were incubated with the appropriate secondary antibody and/or Hoechst 33258 (1:100-1000; Sigma).

The neuronal phenotypes of PDGF-AA-generated progeny were examined in 6 DIV neurospheres differentiated in 1\% FBS for 2 DIV, and E14 dissociated whole brains plated on poly-L-ornithine-coated coverslips for 5 DIV in the presence of $1 \%$ FBS served as a control. Coverslips were incubated overnight at $4^{\circ} \mathrm{C}$ in rabbit anti-rat parvalbumin $(1: 1000$; Swant, Bellinzona, Switzerland), rabbit anti-mouse GABA (1:500; Sigma), rabbit anti-mouse calretinin (1:1000; Swant), mouse anti-mouse calbindin-D (1:200; Sigma), and/or mouse anti- $\beta$-III-tubulin. Coverslips were then incubated with appropriate secondary antibodies as above.

For immunohistochemistry on cryosections, E14 brains were dissected out and processed as described previously (Shimazaki et al., 2001). For staining with rabbit anti-mouse PDGFR $\alpha$ (Santa Cruz Biotechnology), transverse sections $(10 \mu \mathrm{m})$ were first incubated in $1 \% \mathrm{H}_{2} \mathrm{O}_{2}$ in PBS 
for $30 \mathrm{~min}$ at room temperature. Subsequently, sections were incubated with the antibody (1: 300 ) in $0.3 \%$ Triton X-100 and $10 \%$ normal goat serum in PBS overnight at room temperature. Sections were then washed and incubated with the appropriate HRP-conjugated secondary antibody for $1 \mathrm{hr}$ at room temperature. Sections were then incubated with $3,3^{\prime}$-diaminobenzidine $(1 \times 10 \mathrm{mg}$ tablet in $20 \mathrm{ml}$ of PBS and $10 \mu \mathrm{l}$ of $30 \% \mathrm{H}_{2} \mathrm{O}_{2}$; Sigma) for $10 \mathrm{~min}$ or until the desired intensity of reaction product was reached. For double labeling, E14 brains were fixed as above, and 10-15 $\mu \mathrm{m}$ transverse sections were cut on a vibratome (Leica), mounted onto slides, and incubated with sheep antimouse EGFR (1:50; Biodesign International, Kennebunk, ME) or rabbit anti-mouse FGF receptor-2 (FGFR-2) (1:50; Santa Cruz Biotechnology) in PBS. This was followed by washes and incubation with the appropriate biotin-conjugated secondary antibodies for 2 $\mathrm{hr}$ at room temperature. Sections were then washed and incubated with streptavadin-Cy3 ( $1: 1000)$, followed by a $2 \mathrm{hr}$ incubation at $37^{\circ} \mathrm{C}$ with goat anti-mouse PDGFR $\alpha$ (1:10; R \& D Systems) in $0.3 \%$ Triton X-100 in PBS. After washes in PBS, sections were incubated for $1 \mathrm{hr}$ with the appropriate secondary antibody. All immunofluorescent slides were mounted with Fluorsave (Calbiochem, San Diego, CA). Images were captured with a Photometrics Quantix camera or an Axiocam (Zeiss, Thornwood, NY) mounted on a Zeiss Axioplan2.

\section{Results}

\section{The E14 MGE is the source of} neurosphere-generating PRPs with the potential to differentiate into neurons and oligodendrocytes

Given that PDGFR $\alpha$ is one of the earliest markers of OLPs and that signaling by PDGF-AA is required for the generation of most oligodendrocytes (Fruttiger et al., 1999), we first asked whether stimulation of dissociated E14 MGEs and LGEs with increasing concentrations of PDGF-AA would result in the generation of neurospheres. The neurosphere assay was used because manipulation of the primary cells is minimal compared with the immunopanning procedures used to isolate $\mathrm{O}-2 \mathrm{~A}$ progenitors. PDGF-AA induced neurosphere production in a dose-dependent manner with significantly more neurospheres being produced in $100 \mathrm{ng} / \mathrm{ml}$ PDGF-AA compared with all other concentrations tested $[p<$ 0.01; $12 \pm 1$ neurospheres per 10,000 plated cells; Tukey honest significant difference (HSD); $n=3$ ] (Fig. 1A).

Expression of PDGFR $\alpha$ is mainly restricted to the MGE at E14 (Tekki-Kessaris et al., 2001). Therefore, we reasoned that if endogenous PRPs were being isolated, then their generation should be restricted to the MGE. As predicted, the MGE produced significantly more neurospheres (more than fourfold) (Fig. $1 B$ ) than the LGE with either PDGF-AA or PDGF-BB $(p<0.0001 ; t$ test; $n=4$ and $n=3$, respectively), suggesting that we were, in fact, isolating endogenously generated PRPs. PDGF is also known
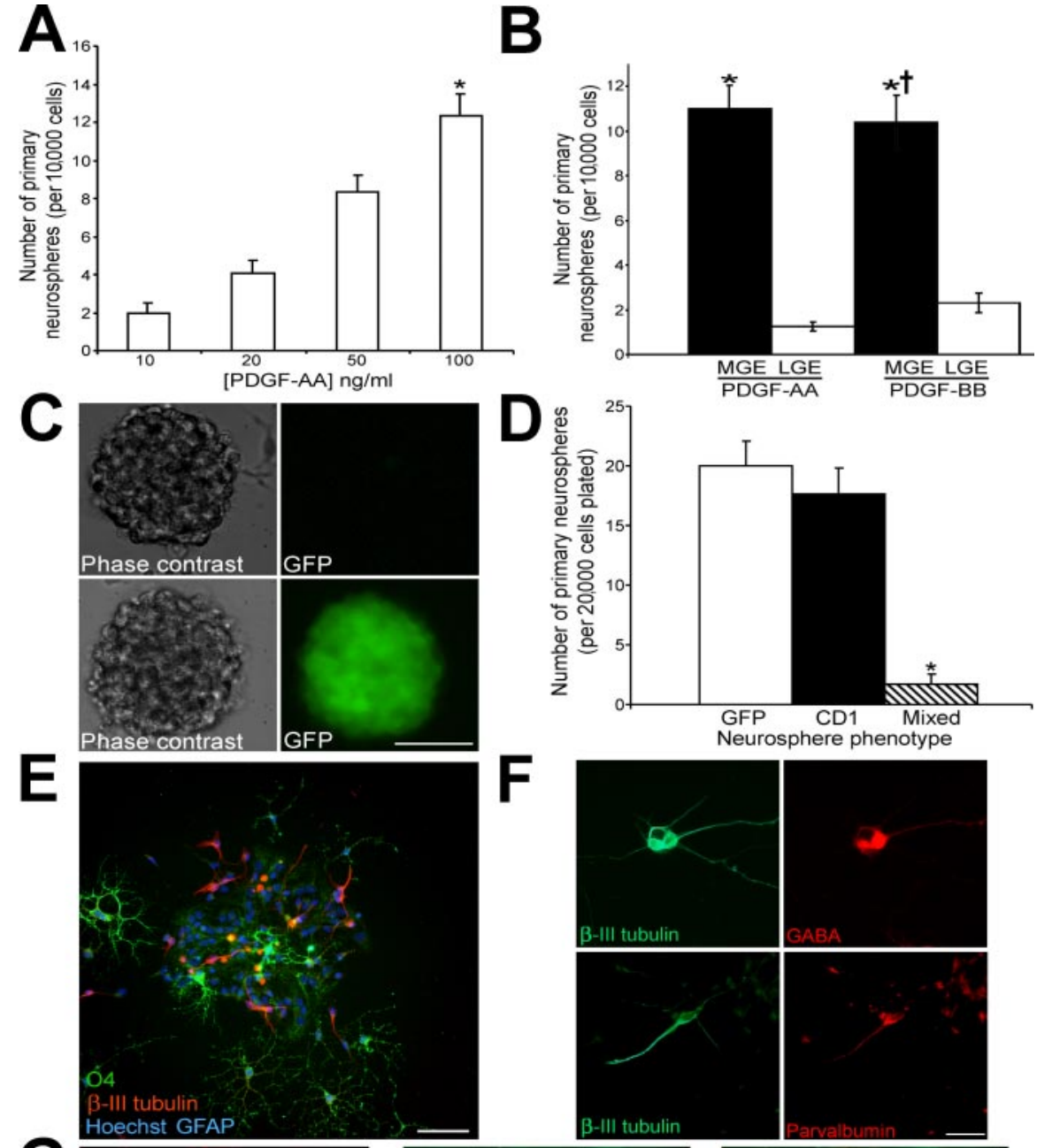

Figure 1. PDGF induces the proliferation of precursors from the MGE with the potential to differentiate into neurons and oligodendrocytes. $A$, PDGF-AA induces the generation of neurospheres from the MGE in a dose-dependent manner. $B$, Significantly more neurospheres were generated from the MGE than the LGE by either PDGF-AA ( ${ }^{*} p<0.0001 ; t$ test; $\left.n=4\right)$ or PDGF-BB $\left({ }^{* \dagger} p<\right.$ $0.0001 ; t$ test; $n=3)$. C, $D$, When GFP- and non-GFP-expressing dissociated E14 MGEs were grown 1:1 for 6 DIV, the vast majority of neurospheres generated were not chimeric for GFP expression ( ${ }^{*} p<0.01$; Tukey HSD; $n=3$ ), suggesting a predominantly clonal proliferation of PRPs. When 6-d-old PDGF-generated neurospheres were differentiated for 2 DIV in 1\% FBS, oligodendrocytes $(E)$ and parvalbumin-immunoreactive GABAergic interneurons $(E, F)$ were detected by immunocytochemistry. $G$, Photomicrographs demonstrating that some PDGFR $\alpha$-expressing precursor cells coexpress the neuron-specific antigen TOAD-64 within the E14 forebrain. Each arrow indicates a cell that is immunoreactive for TOAD-64 and PDGFR $\alpha$. Scale bars: C, E, $50 \mu \mathrm{m} ; F, G, 25 \mu \mathrm{m}$.

to have chemotaxic effects on cortical NSCs (Forsberg-Nilsson et al., 1998), and thus it was possible that neurospheres generated under PDGF stimulation resulted from the directed migration of cells along the culture dish into clumps that resembled clonally derived neurospheres. This was tested, as we have previously done (Represa et al., 2001), by culturing dissociated E14 MGEs from CD-1 and $\operatorname{TgN}(\mathrm{GFPU}) 5 \mathrm{Nagy}$ mice together (1:1) at 20,000 cells/ml and in the presence of $100 \mathrm{ng} / \mathrm{ml}$ PDGF-AA. If cell clumping generates the majority of the neurospheres, then most neurospheres should contain both GFP- and non-GFPexpressing cells. However, $95 \pm 11 \%$ of the neurospheres were 
not chimeric for GFP expression ( $p<0.01$; Tukey HSD; $n=3$ ) (Fig. $1 C, D$ ), and there was no difference in the number of GFP- or non-GFP-expressing neurospheres produced ( $p>0.65$; Tukey HSD; $n=3$ ) (Fig. 1D). Together, the data suggest that neurospheres generated by PDGF stimulation are products of clonal cell proliferation.

Next, the phenotype potential of the PDGF-generated neurospheres was examined with indirect immunocytochemistry using antibodies directed against GFAP (astrocytes), $\beta$-III-tubulin (neurons), and O4 (oligodendrocytes). Surprisingly, PDGFgenerated neurospheres differentiated into neurons and/or oligodendrocytes but not astrocytes after 2 DIV in the presence of $1 \%$ FBS (Fig. $1 E$ ). The MGE primarily gives rise to interneurons that migrate out toward the cortex, in a manner similar to OLPs (Marin and Rubenstein, 2001). Therefore, we asked whether PDGF-generated neurons, which are MGE derived, expressed interneuronal antigens. Immunocytochemistry, with antibodies directed against GABA, calbindin D, calretinin, and parvalbumin, was used to examine the phenotype of the neurons that had differentiated (after 2 DIV in the presence of 1\% FBS) from 6 DIV PDGF-AA-generated neurospheres. All these antigens could be detected in E14 dissociated whole brains differentiated for 5 DIV (data not shown). However, differentiated neurons from PDGFAA-generated neurospheres, identified by $\beta$-III-tubulin immunoreactivity, expressed only GABA or parvalbumin (Fig. $1 F$ ). These findings are in agreement with transplantation studies by Wichterle et al. (2001), which demonstrated that over $70 \%$ of the neurons derived from the MGE differentiated into parvalbuminimmunoreactive, GABAergic interneurons.

Although neurons clearly differentiated from the progeny of PRPs, this could reflect a potential that does not occur in vivo. Thus, we asked whether PRPs coexpress any neuronal antigens in vivo. We found examples of PDGFR $\alpha$-immunoreactive cells within the E14 MGE that were also immunopositive for TOAD64/TUC-2 (Minturn et al., 1995), an early neuron-specific antigen (Fig. 1G). Together, the data suggest that PRPs contribute, in addition to oligodendrocytes, to the generation of neurons within the forebrain.

\section{PRPs are distinct from EGF-responsive NSCs}

The observations that PRPs reside mainly in the MGE and that they do not produce astrocytes suggested that they are distinct from EGF-responsive NSCs. Indeed, EGF can generate neurospheres from both MGE and LGE, and these neurospheres produce neurons, oligodendrocytes, and astrocytes when differentiated in 1\% FBS (Reynolds and Weiss, 1996). If PDGF and EGF stimulate distinct populations to produce neurospheres, one would predict a predominantly nonoverlapping pattern of PDGFR $\alpha$ and EGFR expression within the MGE or anterior entopeduncular (AEP). Thus, the expression of PDGFR $\alpha$ and EGFR was examined in transverse sections of the E14 forebrain. PDGFR $\alpha$ expression was mainly restricted to the AEP, preoptic area, the primordia of the choroid plexus, and the meninges (Fig. $2 A$ ). Double labeling for PDGFR $\alpha$ (arrowhead) and EGFR (arrow) revealed two populations of cells that were nonoverlapping in their expression of these receptors (Fig. $2 B--D$ ). Tropepe et al. (1999) found that embryonic EGF and FGF NSCs were two distinct cell populations, by virtue of their generation of neurospheres being additive under clonal conditions. Thus, if PRPs and EGF-responsive NSCs are truly different populations as suggested by the expression patterns of their receptors, the generation of neurospheres with both PDGF-AA and EGF should also be additive. Accordingly, we cultured cells from the LGE or MGE at 10,000 cells $/ \mathrm{ml}$ in the presence of $100 \mathrm{ng} / \mathrm{ml}$ PDGF-AA, 20 $\mathrm{ng} / \mathrm{ml} \mathrm{EGF}$, or both, and the resultant primary neurospheres were counted. Figure $2 E$ demonstrates that dissociated MGEs cultured in the presence of EGF plus PDGF-AA produced significantly more neurospheres than MGEs cultured in either EGF or PDGF-AA alone ( $p<0.05$; LSD test; $n=4)$. In contrast, there was no difference in the number of neurospheres produced from dissociated LGEs cultured in the presence of EGF plus PDGF-AA compared with EGF alone ( $p>0.86$; LSD test; $n=4)$.

We previously demonstrated that single, primary EGFgenerated neurospheres, when dissociated and cultured in EGF, always produce many secondary neurospheres (Reynolds and Weiss, 1996), indicative of their extensive self-renewal capacity. Therefore, we asked whether PRPs have a similarly extensive capacity for self-renewal. Figure $2 F$ illustrates that single, primary PDGF-generated neurospheres produced almost no secondary neurospheres $(1 \pm 1)$ when mechanically dissociated in 96-well plates containing PDGF-AA $[n=7 ; 69$ neurospheres examined (NE)]. Primary PDGF-generated neurospheres passaged into EGF $(n=3 ; 32 \mathrm{NE})$ also produced very few $(3 \pm 1)$ secondary neurospheres. On the other hand, primary EGF-generated neurospheres processed in the same manner but passaged into PDGF-AA $(n=3 ; 24 \mathrm{NE})$ produced $26 \pm 5$ secondary neurospheres. Thus, unlike EGF NSCs, which have the capacity to passage into EGF or PDGF-AA, primary PRPs rarely self-renew in either PDGF-AA or EGF.

To determine whether there were differences in the differentiation of both types of neurospheres, primary 6 DIV EGF- and PDGF-AA-generated neurospheres were plated for $24 \mathrm{hr}$ on polyL-ornithine-coated coverslips. Within $24 \mathrm{hr}$ of plating, PDGFAA-generated progeny migrated great distances (over $300 \mu \mathrm{m}$ in some instances) from the center of neurospheres (Fig. 2G). In contrast, primary EGF-generated progeny rarely migrated away from the center of differentiating neurospheres (Fig. $2 H$ ). Together, these data suggest that PRPs are a population distinct from that of EGF-responsive NSCs.

\section{BMP-2 and T3 promote the differentiation of PRP-generated neurons and oligodendrocytes, respectively}

$\mathrm{BMP}$ and $\mathrm{T} 3$ can direct the astroglial and oligodendroglial differentiation of O-2A progenitors, respectively (Ahlgren et al., 1997; Mabie et al., 1997). Therefore, we asked whether BMP-2 and T3 could direct the differentiation of cells within PDGF-generated neurospheres. Six days in vitro primary PDGF-AA-generated neurospheres were differentiated on coverslips for 2 DIV in $1 \%$ FBS, 50 ng/ml BMP-2, 20 ng/ml T3, or T3 plus BMP-2. Indirect immunocytochemistry revealed that in the presence of $1 \%$ FBS, $\sim 30 \%$ of the cells differentiated into $\beta$-III-tubulin-expressing neurons, whereas $5 \%$ became $\mathrm{O} 4$ expressing; the remainder of the cells did not express either antigen (Fig. $3 A, E$ ). Compared with control experiments in $1 \% \mathrm{FBS}(n=3 ; 23 \mathrm{NE}), \mathrm{BMP}-2$ had no significant effect on the number $(p>0.15$; Tukey HSD; $n=4$; $38 \mathrm{NE}$ ) of oligodendrocytes produced per clone (Fig. $3 B$ ). However, BMP-2 clearly increased neurite length compared with $1 \%$ FBS (Fig. 3, compare $A, B$ ). On the other hand, T3 increased (fivefold) the differentiation of oligodendrocytes $(p<0.001$; Tukey HSD; $n=4 ; 24 \mathrm{NE}$ ) (Fig. 3, compare $A, C$; ). In the presence of both factors, BMP-2 $(n=4 ; 38 \mathrm{NE})$ suppressed T3induced oligodendrocyte differentiation of PDGF-generated progeny ( $p<0.001$; T3 vs T3 plus BMP-2; Tukey HSD), and neuronal numbers were equivalent to those observed in differentiation with BMP-2 alone ( $p>0.99$; Tukey HSD) (Fig. 3, compare $C, D ; E)$. In all cases, the numbers of differentiated cells 
(10-13 neurons and/or oligodendrocytes) remained constant at approximately onethird of the total clone size $(30-35 \%)$. We did not observe GFAP-immunoreactive cells in any of these culture conditions.

Surprisingly, we occasionally observed cells with an oligodendroglial morphology that expressed $\mathrm{O} 4$ and an inner ring of $\beta$-III-tubulin (Fig. $3 F$ ), but only when differentiated in FBS. Although these early neuronal and oligodendroglial antigens may not definitively identify bona fide neurons and oligodendrocytes, it is more likely that T3 and BMP-2 direct the fate choices of uncommitted PRPs. Therefore, we asked whether BMP-2 and T3 action would result in the expression of more mature neuronal and oligodendroglial antigens, respectively, in differentiating primary PDGF-generated neurospheres. PDGF-generated neurospheres differentiated for 2 DIV expressed NFM (neurons) or MBP (oligodendrocytes), but both were never observed in the same cell, regardless of the differentiation conditions (Fig. $4 A$ ). BMP-2 $(n=3 ; 29 \mathrm{NE})$ increased the percentage of clones expressing NFM compared with differentiation in 1\% FBS $(p<0.05$; Tukey HSD; $n=3 ; 22 \mathrm{NE})$, whereas T3 $(n=3 ; 26 \mathrm{NE})$ increased the number of MBP-only clones compared with $1 \%$ FBS, BMP-2, or T3 plus BMP-2 $(p<0.001$; Tukey HSD; $n=3 ; 27 \mathrm{NE})$ (Fig. 4B). In contrast, BMP-2, when present with $\mathrm{T} 3$, completely inhibited the generation of MBP-only clones $(p<$ 0.001; T3 vs T3 plus BMP-2; Tukey HSD) (Fig. $4 B)$. T3 $(n=4 ; 29 \mathrm{NE})$ alone significantly increased the number of MBPexpressing cells produced per clone compared with $1 \% \mathrm{FBS}(n=4 ; 25 \mathrm{NE}), \mathrm{BMP}-2$ $(n=4 ; 27 \mathrm{NE})$, and T3 plus BMP-2 $(p<$ 0.001 ; T3 vs $1 \%$ FBS, BMP-2, and T3 plus BMP-2; Tukey HSD; $n=4 ; 28 \mathrm{NE}$ ) (Fig. $4 C)$. These data suggest that BMP-2 suppresses oligodendroglial differentiation but promotes neuronal maturation, whereas T3 promotes the maturation of oligodendrocytes from PDGF-generated neurospheres.

BMP-2 together with CNTF suppresses the expression of OLIG2 and promotes astroglial differentiation in a population distinct from that capable of differentiating into neurons and oligodendrocytes We were surprised that GFAP-immunoreactive astrocytes were absent in PDGFgenerated neurospheres differentiated in BMP-2, given previous reports that BMPs induce astrocyte differentiation of O-2A progenitors in vitro (Mabie et al., 1997) and glial progenitors in vivo (Gomes et al., 2003). Therefore, we reexamined the potential of
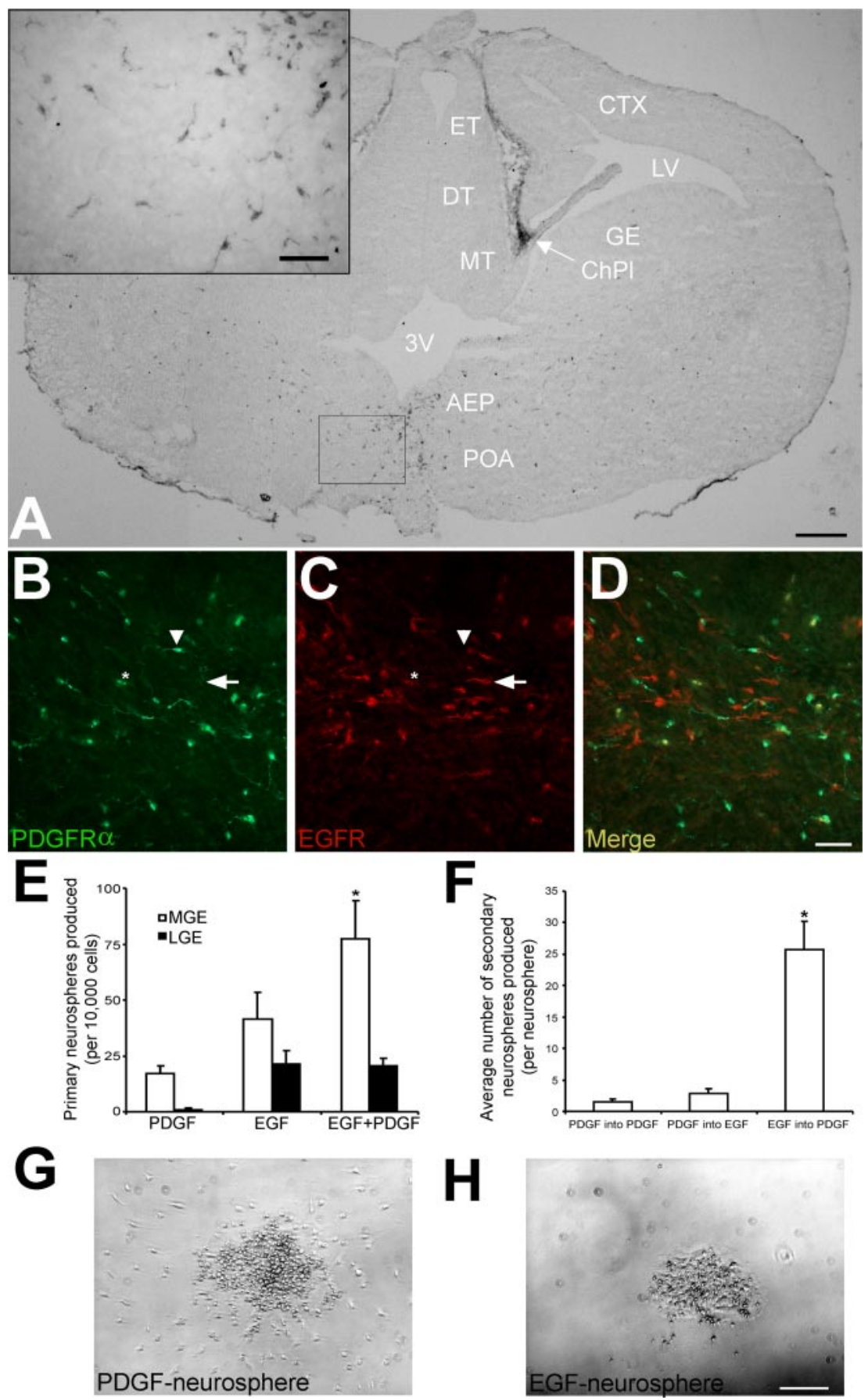

Figure 2. PRPs are distinct from EGF-responsive NSCs. A, Low-power photomicrograph showing PDGFR $\alpha$ immunoreactivity within the AEP (inset) as well as in the primordium of the choroid plexus (arrow). High-power photomicrographs reveal that PDGFR $\alpha$-expressing $(B)$ and EGFR-expressing ( $C$ precursors are separate populations $(D)$. The asterisks in $B$ and $C$ indicate autofluorescent blood cells. $E$, Significantly greater numbers of neurospheres were generated from the MGE in the presence of PDGF-AA plus EGF compared with PDGF-AA or EGF alone ( $p<0.05$; LSD test; $n=4)$. F, PRPs have a limited self-renewal capacity as shown when single neurospheres of the same size were passaged into PDGF ( $n=7 ; 69 \mathrm{NE})$ or EGF $(n=3 ; 32 \mathrm{NE})$ compared with EGF-generated neurospheres passaged into PDGF ( ${ }^{*} p<0.0001$; Tukey HSD; $n=3 ; 24$ NE). PDGF-generated progeny differentiated for $24 \mathrm{hr}$ migrated large distances away (some $>300 \mu \mathrm{m}$ ) from the center of differentiating neurospheres $(G)$ compared with EGF-generated neurospheres $(H)$ that appeared to flatten out, and individual cells were rarely seen migrating away from the neurospheres. Scale bars: $A, 200 \mu \mathrm{m}$; inset in $A, D, 50 \mu \mathrm{m} ; H, 100 \mu \mathrm{m}$.

PDGF-generated progeny to differentiate into astrocytes in the presence of CNTF, another factor known to induce astrocyte differentiation of O-2A progenitors (Hughes et al., 1988). We found that CNTF on its own did not induce GFAP expression in PDGF-generated progeny (Fig. 5A). It has been previously shown 

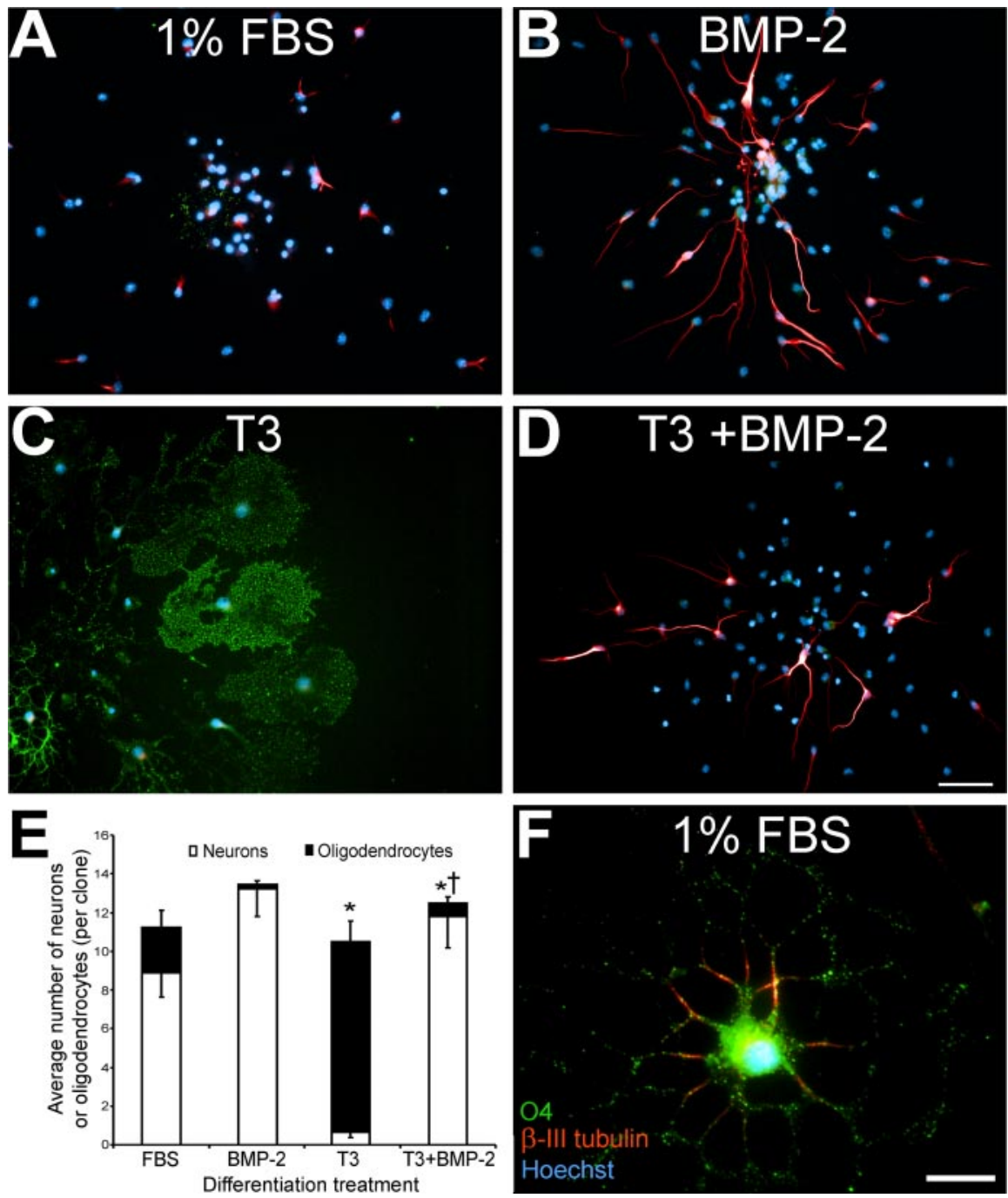

Figure 3. BMP-2 and T3 promote the differentiation of neurons and oligodendrocytes from PDGF-generated neurospheres, respectively. Six days in vitro primary PDGF-AA-generated neurospheres differentiated for $2 \mathrm{DIV}$ in the presence of $1 \% \mathrm{FBS}(A ; n=$ $3 ; 23 \mathrm{NE}), 50 \mathrm{ng} / \mathrm{ml} \mathrm{BMP}-2$ ( $B ; n=4 ; 38 \mathrm{NE}), 20 \mathrm{ng} / \mathrm{ml} \mathrm{T3}(C ; n=4 ; 24 \mathrm{NE})$, or T3 plus BMP-2 ( $; ; n=4 ; 25 \mathrm{NE})$ were processed for immunocytochemistry against $\beta$-III-tubulin (neurons), 04 (oligodendrocytes), and Hoechst (nuclei; blue), and the number of immunoreactive cells was counted $(E)$. Treatment of neurospheres with $T 3(C, E)$ promoted oligodendrocyte differentiation $\left({ }^{*} p<\right.$ 0.001; Tukey HSD), whereas BMP-2 suppressed T3-induced oligodendrocyte differentiation and promoted neuronal differentiation $\left(D, E_{*}^{* \dagger} p<0.001\right.$; Tukey HSD). Occasionally, but only in the presence of $1 \% \mathrm{FBS}$, cells were observed with an oligodendroglial morphology that expressed both 04 and $\beta$-III-tubulin. Scale bars: D, $50 \mu \mathrm{m} ; F, 12.5 \mu \mathrm{m}$.

that leukemia inhibitory factor and BMP signaling can synergize to induce astrocyte differentiation of fetal neural progenitors (Nakashima et al., 1999). Therefore, we asked whether such synergistic signaling may also reveal astrocyte differentiation in PDGF-generated neurospheres. When PDGF-generated progeny were differentiated in the presence of BMP-2 and CNTF, a large number of GFAP-immunoreactive cells with astrocyte morphology were apparent (Fig. 5A). Indeed, the PDGF-generated neurospheres differentiated into neurons and astrocytes but not oligodendrocytes in the presence of BMP-2 and CNTF (Fig. 5B). In addition, we found that the numbers of neurons that differentiated in the presence of BMP-2 plus CNTF $(8 \pm 1 ; 24 \mathrm{NE})$ were not significantly different from those seen with either BMP-2 alone or BMP-2 plus T3. In contrast, the numbers of undifferentiated cells were dramatically reduced from $65-70 \%$ in FBS, BMP-2, T3, and BMP-2 plus T3 conditions to 2-4\% (24 NE) in the presence of
BMP-2 plus CNTF. Thus, PRP progeny have the potential to differentiate into astrocytes.

The findings above suggest that the PRP progeny that differentiate into astrocytes in the presence of BMP-2 plus CNTF may be a population of cells distinct from the neuron or oligodendrocyte (N/O) cells. If this were the case, then one would predict that PDGF-generated neurospheres treated with T3, followed by BMP-2 plus CNTF, should yield clones that contain both oligodendrocytes and astrocytes. Thus, we differentiated 6 DIV neurospheres in the presence of $\mathrm{T} 3$ for 3 DIV or in the presence of T3 with BMP-2 plus $\mathrm{CNTF}$ added after the second day. We delayed the addition of BMP-2 plus CNTF by $2 \mathrm{~d}$ to ensure the oligodendrocytes had been specified by the N/O cells and to prevent their predominant differentiation into neurons by BMP-2. Neurospheres that had BMP-2 plus CNTF added to them after 2 DIV in T3 contained both MBP-immunoreactive oligodendrocytes (4.9 $\pm 0.7 ; n=3 ; 34 \mathrm{NE})$ and GFAPimmunoreactive astrocytes $(2.4 \pm 0.4)$, in contrast to cultures differentiated in $\mathrm{T} 3$ that contained oligodendrocytes (3.9 \pm 0.4 ) but no astrocytes (Fig. $5 C, D$ ). Furthermore, the number of oligodendrocytes was not reduced when BMP-2 plus $\mathrm{CNTF}$ were added, suggesting that BMP-2 plus CNTF does not promote the differentiation of astrocytes from cells capable of oligodendrocyte differentiation.

It has been previously reported that BMP-2 overexpression in the chick spinal cord decreased the expression of OLIG2 and oligodendrocyte specification (Mekki-Dauriac et al., 2002). More recently, OLIG2 has also been found to directly suppress the astrocyte differentiation pathway (Fukuda et al., 2004). Thus, we predicted that OLIG2 expression might also be suppressed by BMP-2 and perhaps by BMP-2 and CNTF in PDGFgenerated progeny. We examined the expression of OLIG2 and O4 by indirect immunocytochemistry after 6 DIV PDGFgenerated neurospheres had been differentiated for 2 DIV (Fig. $5 E, F)$. BMP-2 significantly reduced the expression of OLIG2 in PDGF-generated progeny compared with MHM, 1\% FBS, T3, T3 plus BMP-2, and CNTF. However, OLIG2 expression was still seen in cultures differentiated in BMP-2, albeit in fewer cells and at a reduced level. Although CNTF had no effect on OLIG2 or O4 expression on its own, when combined with BMP-2, PDGF neurospheres lost virtually all OLIG2 and O4 expression (Fig. 5E,F). However, loss of OLIG2 expression alone cannot account for the induction of astroglial differentiation by BMP-2 plus CNTF, considering that in the other differentiation conditions astrocytes did not emerge although a substantial number of cells did not express OLIG2. These data suggest that BMP-2 alone reduces OLIG2 expression, which may suppress oligodendrocyte differentiation 

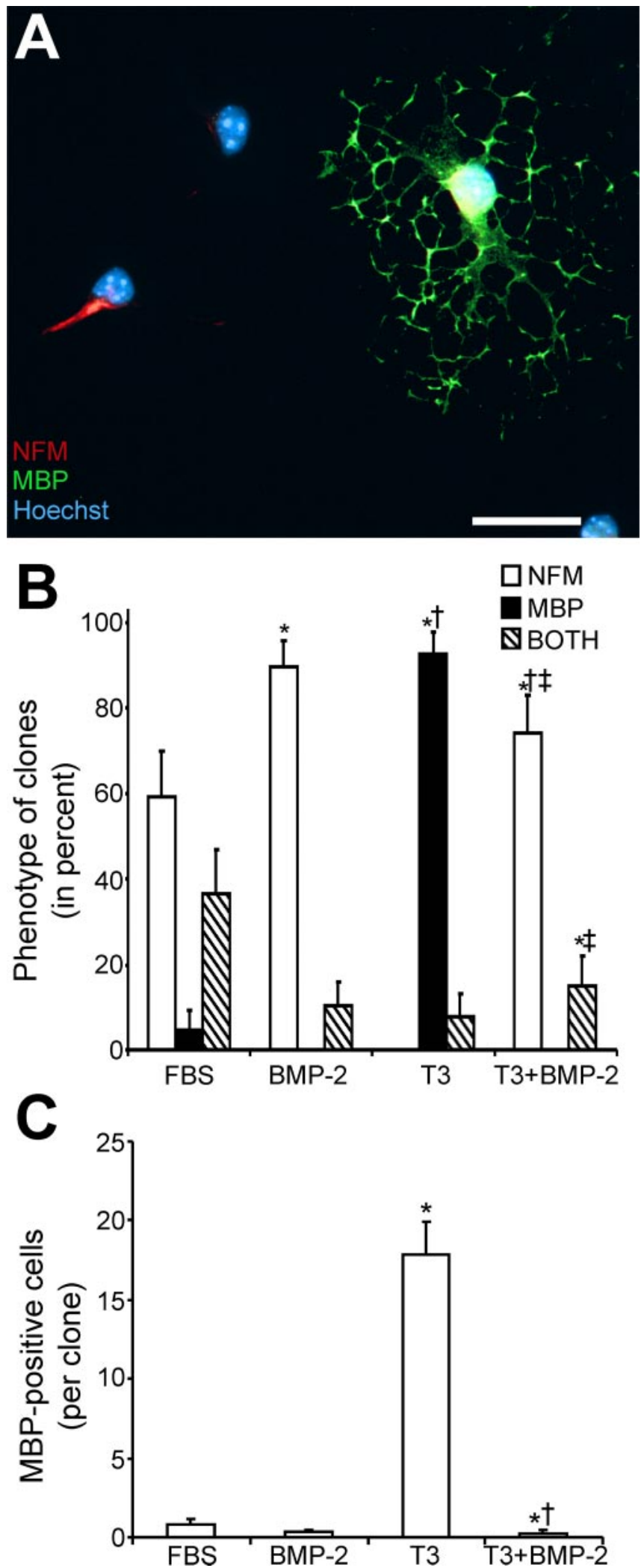

Figure 4. T3 promotes and BMP-2 inhibits the expression of mature oligodendroglial antigens in differentiating, primary PDGF-generated neurospheres. $A$, Photomicrograph of MBP and NFM immunoreactivity as well as Hoechst nuclear staining in PDGF-generated neurospheres differentiated in 1\% FBS. Scale bar, $12.5 \mu \mathrm{m}$. B, BMP-2 ( $n=3 ; 29 \mathrm{NE}$ ) increased the percentage of clones expressing NFM compared with differentiation in $1 \%$ FBS $\left({ }^{*} p<0.05\right.$; Tukey HSD; $n=3 ; 22 \mathrm{NE}$ ). In contrast, T3 ( $n=3 ; 26 \mathrm{NE})$ promoted oligodendrocyte maturation ( ${ }^{*} p<0.001$ vs $1 \%$ FBS; Tukey HSD). Compared with T3 alone, BMP-2 suppressed the actions of T3 on oligodendroglial differentiation $\left({ }^{* *} p<0.001\right.$; Tukey HSD; $n=3 ; 27 \mathrm{NE}$ ) and promoted and promote neuronal differentiation, whereas together BMP-2 and CNTF signaling further reduce levels of OLIG2 expression in PDGF-generated progeny and promote astrocyte differentiation.

\section{Generation of neurospheres by PDGF depends on SHH signaling}

Signaling by SHH is necessary for the generation of OLPs in the mammalian forebrain (Nery et al., 2001; Tekki-Kessaris et al., 2001). We hypothesized that the proliferation of PRPs may be sensitive to $\mathrm{SHH}$ signaling and used loss- and gain-of-function studies to examine its effects. We examined the actions of $\mathrm{SHH}$ signaling on PRPs by growing dissociated MGEs at 10,000 cells/ml in $100 \mathrm{ng} / \mathrm{ml}$ PDGF-AA alone or together with $5 \mu \mathrm{M}$ cyclopamine, a known inhibitor of $\mathrm{SHH}$ signaling (Cooper et al., 1998; Taipale et al., 2000). Neurospheres generated in the presence of cyclopamine were smaller than those generated in PDGF-AA plus DMSO controls (Fig. 6, compare A, B). Cyclopamine also reduced the number of PDGF-AA-generated neurospheres by fivefold ( $p<0.003 ; t$ test; $n=3$ ) (Fig. $6 C$ ). There were large numbers of phase-bright differentiating cells in both conditions (Fig. 6B, arrows), suggesting that the decrease in neurosphere numbers and size is likely not a result of a nonspecific toxic effect.

Because inhibition of SHH signaling attenuated the proliferation of PRPs, we next asked whether increasing $\mathrm{SHH}$ signaling could augment the number of neurospheres generated in the presence of PDGF. Primary cells from dissociated MGEs were cultured in the presence of PDGF-AA, $2 \mu \mathrm{g} / \mathrm{ml}$ of the $19 \mathrm{kDa}$ $\mathrm{N}$-terminal fragment of $\mathrm{SHH}$, or in the presence of both factors. Figure $6 D$ shows that although $\mathrm{SHH}(n=3)$ had no effect on its own, significantly more neurospheres ( $p<0.05$; LSD test) were generated in the presence of SHH plus PDGF-AA $(92 \pm 14 ; n=$ 8) compared with PDGF-AA (60 $\pm 9 ; n=8)$. Together, these data suggest that $\mathrm{SHH}$ is required in concert with $\mathrm{PDGF}$ signaling for the proliferation and/or survival of PRPs.

\section{Self-renewal of PRPs is dependent on growth factor-dependent $\mathrm{SHH}$ signaling}

Although initial experiments suggested that PRPs lacked significant self-renewal capacity (Fig. $2 F$ ), it was plausible that because of our use of defined culture media, some factors required for self-renewal were missing. To test this, we grew primary PDGF neurospheres from the E14 MGEs of TgN(GFPU)5Nagy mice for 7 DIV, dissociated individual neurospheres, and transferred dissociates from single neurospheres onto poly-L-ornithine-coated coverslips that had been plated with or without 200,000 primary 7 DIV EGF-generated progeny $2 \mathrm{~d}$ earlier (see Materials and Methods). In the absence of the EGF-generated feeder layer or PDGF, large adherent colonies of GFP-positive cells were rarely observed (Fig. 7A). In fact, most clones consisted of fewer than 10 cells (Fig. 7C). In contrast, single dissociates plated in the presence of PDGF and the feeder layer produced many clones of GFP-labeled cells that contained $>10$ cells (Fig. $7 \mathrm{~B}, \mathrm{C}$ ), and many of these cells were found to express PDGFR $\alpha$ (Fig. 7D). When single dissociates were plated on the feeder layer but in the absence of PDGF, few clones were observed, and all were $<10$ cells

\section{$\leftarrow$}

the percentage of NFM-only clones ( ${ }^{*+\neq} p<0.001$; Tukey HSD). Cells immunoreactive for both NFM and MBP were not observed. C, Compared with $1 \%$ FBS ( $n=4 ; 25 \mathrm{NE})$ and BMP-2 ( $n=4$; $27 \mathrm{NE})$ treatment, T3 increased the number of MBP-expressing cells per clone $\left({ }^{*} p<0.001\right.$; Tukey HSD; $n=4 ; 29 \mathrm{NE}$ ). The T3-induced increase in MBP immunoreactivity was suppressed by BMP-2 ( ${ }^{*} p<0.001$; Tukey HSD; $\left.n=3 ; 28 \mathrm{NE}\right)$. 

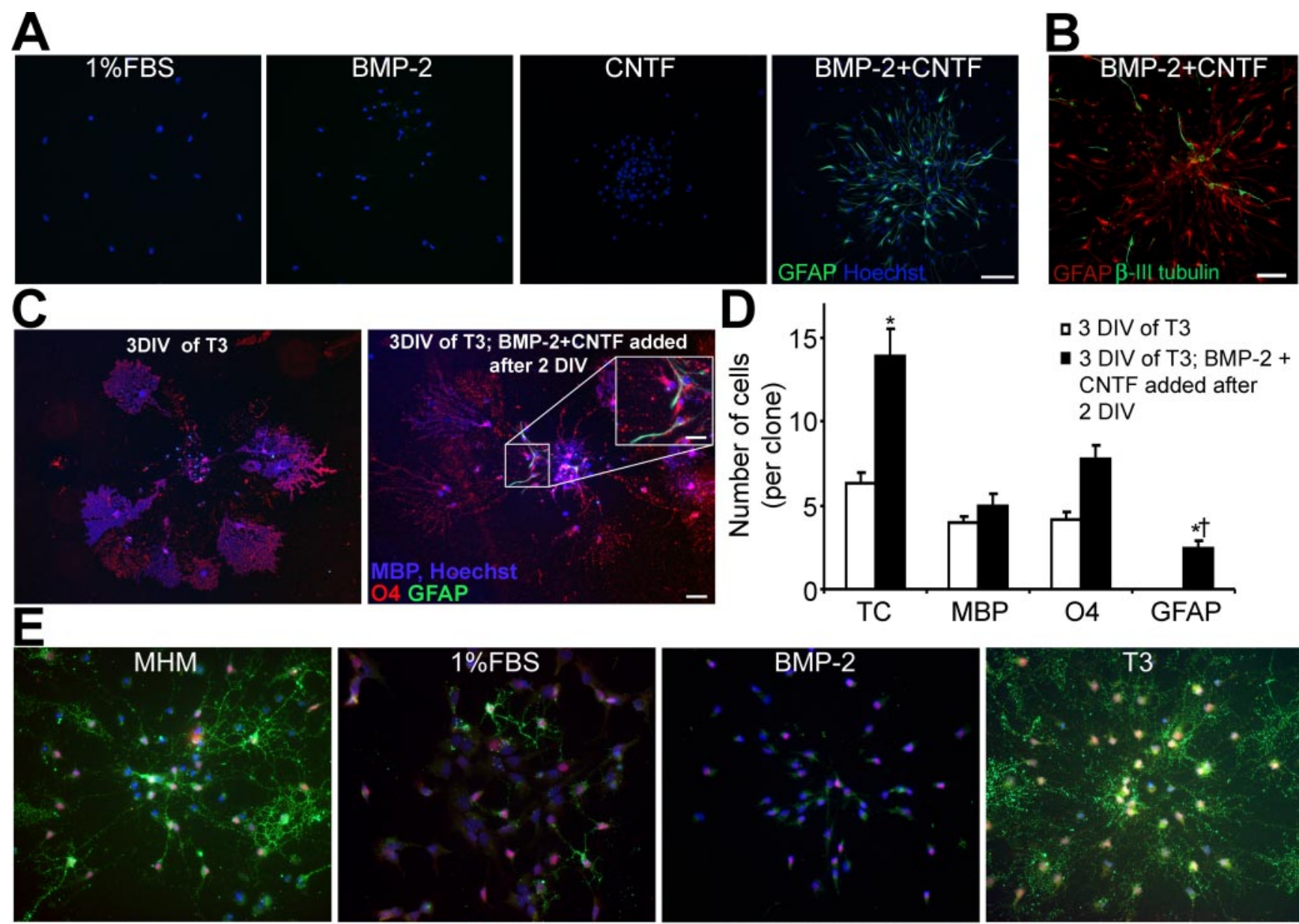

O4 GFAP
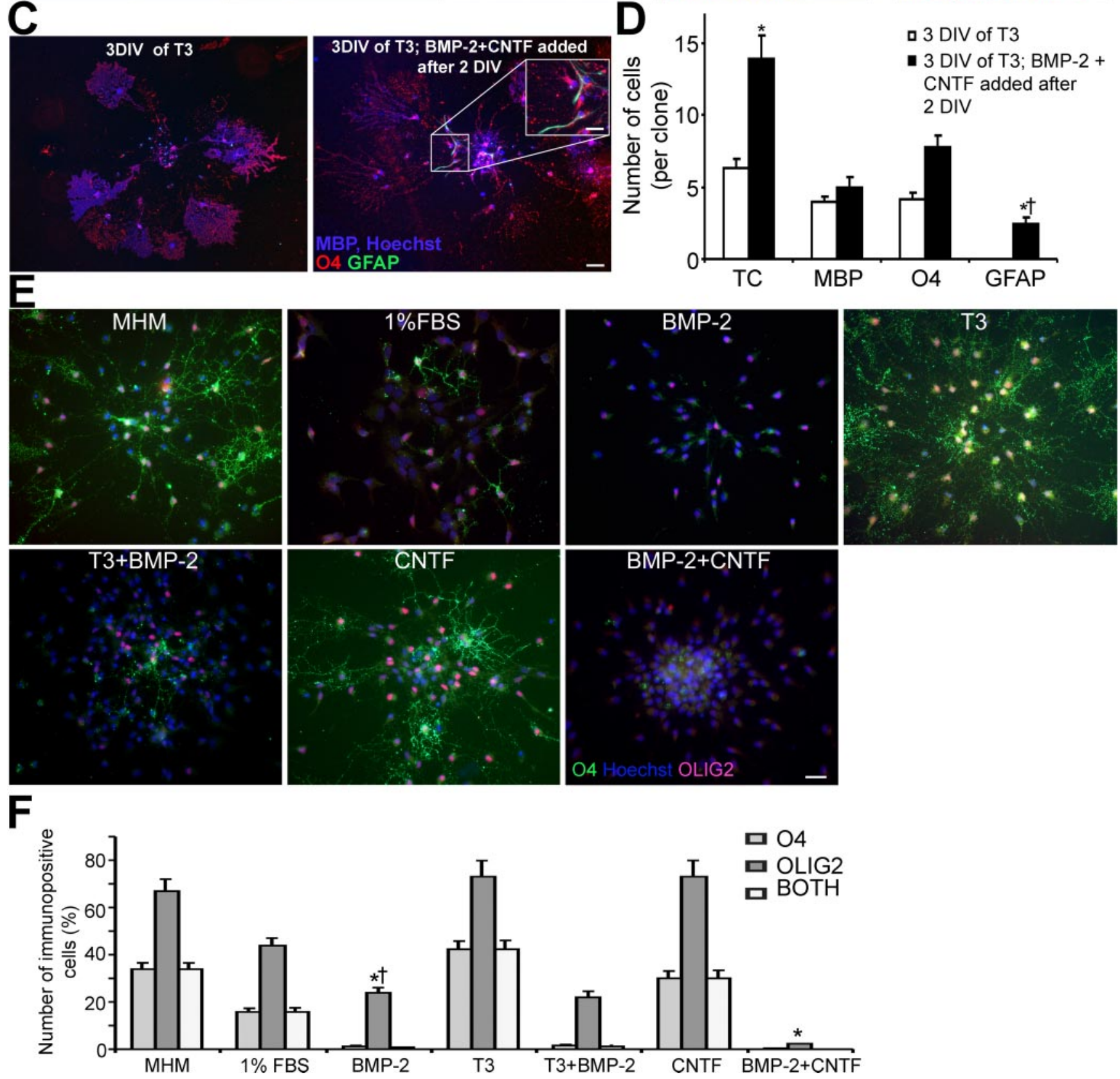

Figure 5. BMP-2 and CNTF promote astroglial differentiation of a distinct population in part by cooperatively suppressing 0LIG2 expression. $A$, Astroglial differentiation of PDGF-generated neurospheres is only evident after a $2 \mathrm{~d}$ treatment with BMP-2 and CNTF. B, Neuronal differentiation of PDGF-generated neurospheres is not suppressed by BMP-2 and CNTF treatment. C, D, Three days of differentiation in T3 ( $n=3 ; 53 \mathrm{NE}$ ) promoted oligodendroglial and not astroglial differentiation in primary PDGF-generated neurospheres. The addition of BMP-2 plus CNTF ( $n=3 ; 34$ NE) after the second day resulted in greater cell survival $\left(D ;{ }^{*} p<0.001 ; t\right.$ test), and a significant number of the undifferentiated cells adopted an astroglial cell fate, but not at the expense of oligodendrocytes $\left(C, D ;{ }^{* \dagger} p<0.0001 ; t\right.$ test), suggesting that BMP-2 and CNTF induce astroglial differentiation in a population of cells separate from those that differentiate into oligodendrocytes. TC, Total cell number. E, F, BMP-2 ( $n=4 ; 56$ NE) can suppress 04 expression as efficaciously as BMP-2 and (NTF in combination ( $p>0.99 ;$ Tukey HSD; $n=4 ; 51$ NE), but together BMP-2 and (NTF are more effective at suppressing 0 LIG2 expression compared with BMP-2 alone $\left({ }^{*} p<0.0001\right.$; Tukey HSD). CNTF ( $n=4 ; 48$ NE) had no effect on 0LIG2 or 04 expression compared with MHM ( $p>$ $0.70 ; n=4 ; 67 \mathrm{NE})$. F shows 1\% FBS ( $n=4 ; 59 \mathrm{NE})$, T3 ( $n=4 ; 45 \mathrm{NE})$, and T3 plus BMP-2 $\left(n=4 ; 43 \mathrm{NE}\right.$; ${ }^{* \dagger} p<0.0001$; Tukey HSD). Scale bars in $A-C, 50 \mu \mathrm{m}$; inset in C, E, $25 \mu \mathrm{m}$. 


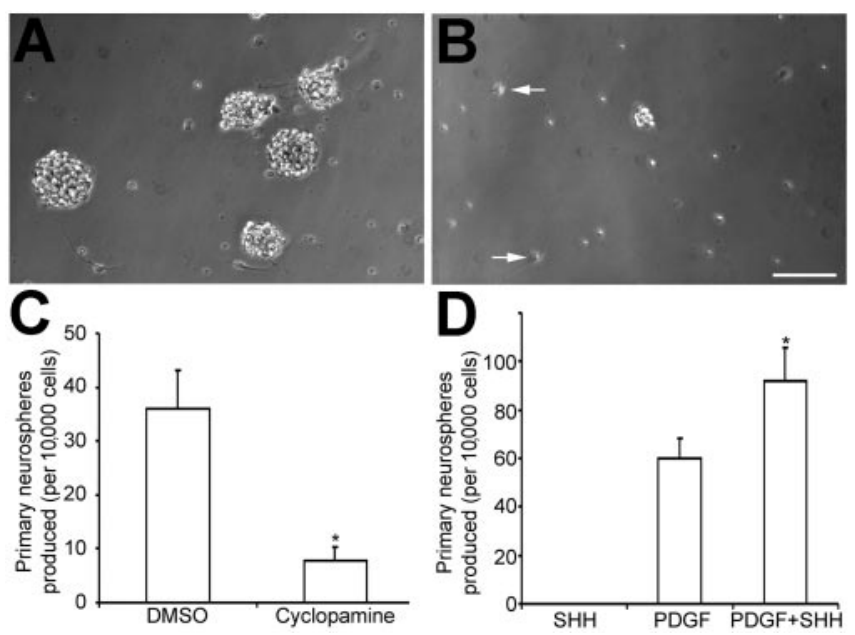

Figure 6. SHH signaling promotes the generation of primary neurospheres by PRPs. PDGFgenerated neurospheres produced in the presence of $5 \mu$ m cyclopamine $(B)$ were clearly smaller than those generated in the presence of DMSO $(A)$, and there was a significant decrease in the numbers produced $\left(C ;{ }^{*} p<0.01 ; t\right.$ test; $\left.n=3\right)$. The arrows in $B$ illustrate differentiating phase-bright cells, which suggests that differentiation proceeds normally in the presence of cyclopamine and that its effects on proliferation or survival of PRPs are not attributable to toxicity. D, SHH signaling significantly enhances the generation of primary PDGF-generated neurospheres compared with PDGF alone $\left({ }^{*} p<0.05\right.$; LSD test; $\left.n=8\right)$, suggesting it promotes the survival/proliferation of PRPs. Scale bar, (in $B$ ) $100 \mu \mathrm{m}$.

in number (Fig. 7C). These results suggest that some signals initiated by the EGF-generated feeder layer, whether soluble or contact dependent, are required for the self-renewal of PRPs together with PDGF.

Because SHH could enhance the number of primary neurospheres generated by PDGF (Fig. 4), we asked whether SHH could also promote the formation of secondary neurospheres from primary PDGF-generated neurospheres. Surprisingly, we found no significant difference ( $p>0.99$; Tukey HSD) in the number of secondary neurospheres generated when primary neurospheres were grown in the presence of PDGF and passaged into PDGF plus $\mathrm{SHH}(n=3 ; 27 \mathrm{NE})$ or grown in the presence of PDGF plus SHH ( $n=4 ; 37 \mathrm{NE})$ and passaged into PDGF compared with those grown and passaged in PDGF $(n=7 ; 69 \mathrm{NE})$ (Fig. 7E). The results suggest that self-renewal, as measured by the number of secondary neurospheres, cannot be augmented by $\mathrm{SHH}$ alone.

Signaling by FGF-2, in combination with PDGF, has previously been shown to promote the self-renewal and inhibit the differentiation of O-2A progenitors in vitro, which normally lose responsiveness to PDGF after several rounds of cell division and differentiate (Bogler et al., 1990). Moreover, a recent study reports that full SHH actions on oligodendrocyte development depends on FGF-2-stimulated mitogen-activated protein kinase (MAPK) activity (Kessaris et al., 2004). To explore these possibilities, we first asked whether precursors that expressed PDGFR $\alpha$ in the ventral forebrain also expressed FGFRs. FGF-2 can bind to the four known FGFRs, FGFRs 1-4 (Reuss and von Bohlen und Halbach, 2003). We found that FGFR-2 immunoreactivity was localized to nuclei in the ventricular zone and to scattered cells within the MGE (Fig. 7F). Some of the FGFR-2-labeled nuclei outside the ventricular zone clearly belonged to cells that expressed PDGFR $\alpha$ (Fig. 7F, arrows), indicating that both signaling pathways may regulate the proliferation and self-renewal of PRPs. We examined whether FGF-2 signaling alone or together with $\mathrm{SHH}$ could, in fact, augment the generation of secondary neurospheres by PRPs. We dissociated individual 6 DIV PDGFgenerated neurospheres and passaged them in media containing either FGF-2 $(n=7 ; 64 \mathrm{NE})$ or FGF-2 plus SHH $(n=7 ; 60 \mathrm{NE})$. No increase in self-renewal was evident in PDGF-generated progeny that had been passaged into FGF-2 compared with PDGF $(p>0.99)$ (Fig. 7, compare $E, G)$. However, in the presence of FGF-2, SHH significantly enhanced the self-renewal of PRPs (Fig. $7 G$ ) compared with primary PDGF-generated neurospheres passaged into either FGF-2 ( $p<0.05$; Tukey HSD) or PDGF $(p>$ 0.01; Tukey HSD) (Fig. 7E). We next asked whether PDGF plus FGF-2 could cooperate in augmenting secondary neurosphere formation. In the presence of PDGF plus FGF-2 $(n=9 ; 78 \mathrm{NE})$, $27 \pm 3$ secondary neurospheres were generated (Fig. $7 G$ ), suggesting that both PDGF and FGF-2 signaling are required for the self-renewal of PRPs, and this was not further increased when SHH was added $(28 \pm 3$ secondary neurospheres; $p>0.99$; Tukey HSD; $n=5 ; 45 \mathrm{NE}$ ). The contention that FGF-2 is merely supporting proliferation of PRPs is not likely the case, given that primary PDGF neurosphere formation was unaffected by $1 \mu \mathrm{M}$ SU5402 (an FGFR tyrosine kinase inhibitor) (Mohammadi et al., 1997), which was able to block $90 \%$ of FGF-2-induced NSC proliferation (data not shown).

Despite the inability of added SHH to augment secondary PDGF neurosphere formation, we hypothesized that the cooperative actions of PDGF plus FGF-2 might be sufficient to support intrinsic SHH signaling, the latter of which is normally necessary for oligodendrocyte generation. Indeed, we found that the selfrenewal of PRPs passaged into PDGF plus FGF-2 was dependent on $\mathrm{SHH}$ signaling, because cyclopamine reduced the generation of secondary neurospheres $(3 \pm 1 ; n=4 ; 36 \mathrm{NE}$ ) (Fig. $7 G)$ to numbers closer to those obtained with PDGF $(1 \pm 1)$ or FGF-2 $(3 \pm 1)$. Together, these findings suggest the full expression of self-renewal capacity by PRPs is dependent on the effective activation of SHH signaling by both PDGF and FGF-2.

\section{Discussion}

The potential of CNS OLPs to also generate neurons and/or astrocytes is a subject of considerable debate (Liu and Rao, 2003; Noble et al., 2004; Rowitch, 2004). Most studies have focused on either the spinal cord or optic nerve, and to our knowledge, none have directly examined the differentiation and self-renewal potential of embryonic ventral forebrain PRPs. We demonstrated that the neurosphere assay is a novel, simple, and useful way of studying factors that regulate the differentiation and self-renewal of PRPs. We found that at E14 PRPs are restricted to the MGE; have the capacity to generate neurons, oligodendrocytes, and astrocytes; and are distinct from EGF-responsive NSCs in several respects. The latter conclusion is supported by the observations that PDGFR $\alpha$ and EGFR are expressed by separate populations within the MGE and that dissociated MGEs cultured in the presence of PDGF plus EGF yields significantly more neurospheres than either factor alone. Furthermore, when differentiated in FBS, the progeny of PRPs differentiate into neurons and oligodendrocytes, whereas EGF-generated progeny differentiate into neurons, oligodendrocytes, and astrocytes; PRPs are not selfrenewing when passaged into EGF or PDGF (whereas EGF NSCs are); and the progeny of PRPs are highly motile compared with EGF-generated progeny.

One of the fundamental findings of this study is that ventral forebrain PRPs have the potential to generate neurons and that these neurons arise from a common neuron/oligodendrocyte precursor that can be induced to undergo neuronal differentiation with BMP-2 and oligodendroglial differentiation with T3. 

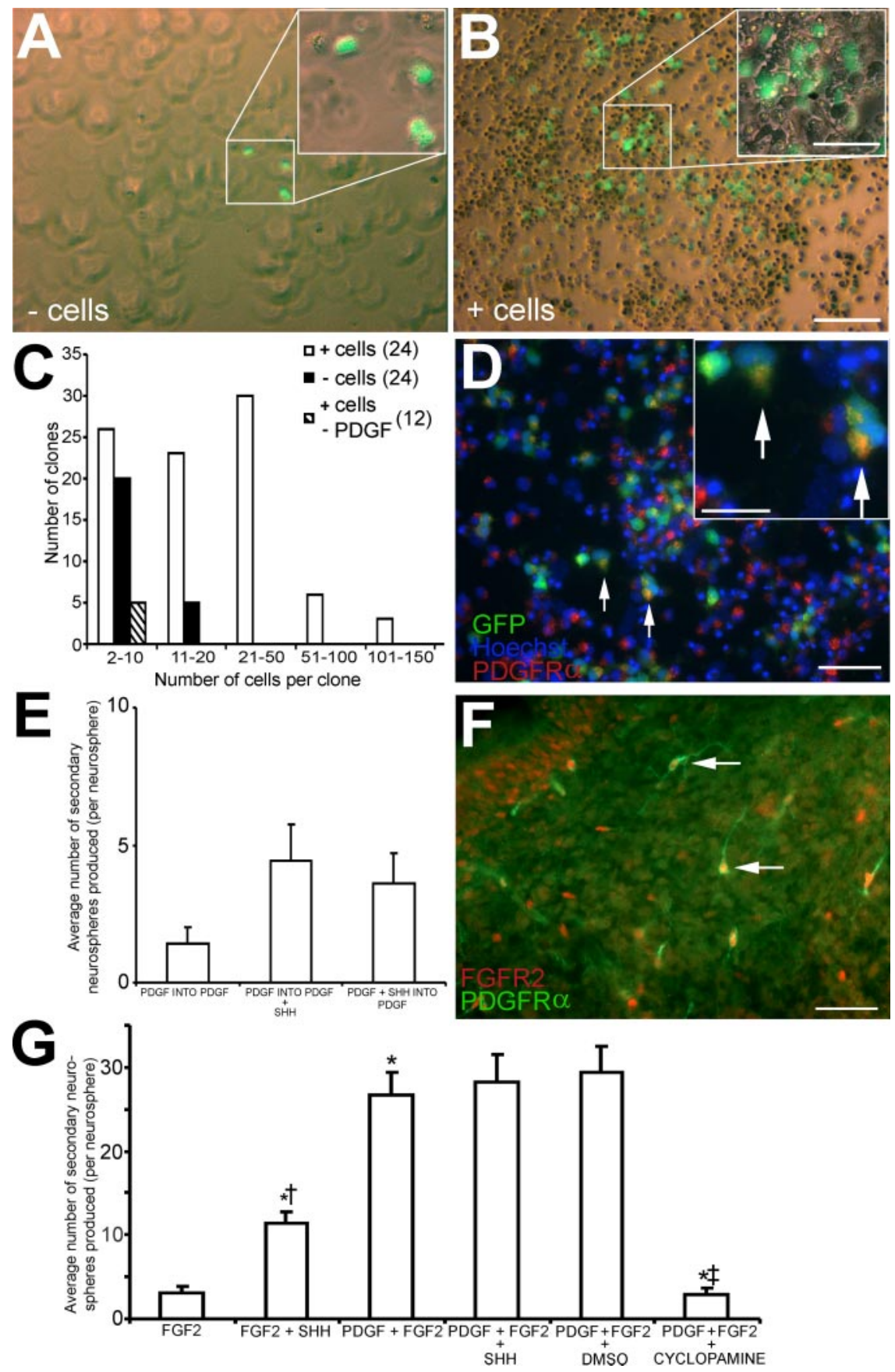

Figure 7. PDGF and FGF-2 signaling cooperatively promote the self-renewal of PRPs through a SHH-dependent pathway. Seven-day-old primary, GFP-expressing, PDGF-generated neurospheres were isolated, dissociated, and plated in the presence of PDGF on poly-L-ornithine-coated coverslips that had been plated $2 \mathrm{~d}$ earlier with $(A)$ or without $(B)$ dissociated, primary EGFgenerated neurospheres differentiated in $1 \%$ FBS. FBS was washed out before dissociates of GFP-expressing, PDGF-generated neurospheres were added. Large adherent clones of GFP-expressing PRPs were generated in the presence of the EGF-feeder layer and PDGF-AA ( $A, C$; numbers in parentheses indicate number of NE) but not in the presence of PDGF alone $(B, C)$ or in the presence of the EGF-feeder layer only $(C$, suggesting that extrinsic factors generated by the EGF-feeder layer could promote the selfrenewal of PRPs in concert with PDGF. D, Photomicrograph demonstrating that some GFP-expressing cells within a clone continue to express PDGFR $\alpha$ (arrows). E, There was no significant increase ( $p>99$; Tukey HSD) in the generation of secondary neurospheres when primary PRPs were grown in conjunction with SHH $(2 \mu \mathrm{g} / \mathrm{ml} ; n=4 ; 37 \mathrm{NE})$ or when passaged into PDGF plus SHH $(n=3 ; 27 \mathrm{NE})$ compared with neurospheres generated and passaged in the presence of PDGF alone ( $n=7 ; 69 \mathrm{NE}) . F$, PDGFR $\alpha-$ expressing cells coexpress FGFR- 2 in the E14 MGE. G, By itself, FGF-2 ( $n=7 ; 64$ NE) had no significant effect on the generation of secondary neurospheres ( $p>0.99$; Tukey HSD) by single, dissociated PDGF-generated neurospheres, but when combined with $\operatorname{PDGF}(n=9 ; 78 \mathrm{NE})$, a significant increase in the generation of secondary neurospheres was observed ( ${ }^{*} p<0.0001$; Tukey HSD). $\mathrm{SHH}(n=7 ; 60 \mathrm{NE})$ also promoted the generation of secondary neurospheres in the presence of FGF-2, but not as robustly as PDGF plus FGF-2 $\left({ }^{* \dagger} p<0.01\right.$; Tukey HSD). The cooperative ability of PDGF and FGF-2 to promote the generation of secondary neurospheres was inhibited by the SHH signaling inhibitor cyclopamine $\left(5 \mu \mathrm{m}^{*{ }^{\ddagger}} p<0.0001\right.$; Tukey HSD; $n=4 ; 36 \mathrm{NE}$; PDGF plus FGF-2 plus DMSO; $n=4 ; 36 \mathrm{NE})$. Scale bars in $B, 100 \mu \mathrm{m}$; inset in $B, D, F, 50 \mu \mathrm{m}$; inset in $D, 25 \mu \mathrm{m}$.
Whether or not the potential of PRPs to generate neurons is manifested in vivo remains to be determined, but several lines of evidence suggest that a common neuron/oligodendrocyte precursor may exist in the developing forebrain. First, we found that, in vivo, a subset of PDGFR $\alpha$-expressing cells coexpress the neuronally restricted antigen TOAD-64. Second, PRPs generate parvalbumin-immunoreactive, GABAergic interneurons, in agreement with transplantation studies that demonstrated that over $70 \%$ of the neurons derived from the MGE become parvalbumin-immunoreactive, GABAergic interneurons (Wichterle et al. 2001). Third, tangential migration of both oligodendrocytes and neurons is disrupted in Dlx1/2 mutant mice, and BMP-2 enhances the generation of pure GABAergic neuronal clones at the expense of mixed neuronal/oligodendroglial clones from premigratory stage MGE or LGE progenitors (Yung et al., 2002). Finally, in vivo (Price and Thurlow, 1988; Grove et al., 1993) and in vitro (Williams et al., 1991), retroviral lineage-tracing studies of the E16 cortex have demonstrated the existence of clones that could generate both neurons and white matter cells or neurons and oligodendrocytes, respectively. Based on the morphology of the white matter cells labeled in these clones, Price and Thurlow (1988) suggested that they were the cerebral equivalent of the $\mathrm{O}-2 \mathrm{~A}$ progenitor. It is likely that the cells labeled were, in fact, PRPs that had migrated from the MGE to the cortex by E16, given our preliminary findings that PRPs are present in the E16 cortex (data not shown). Together, data from the studies of several laboratories suggests that a common precursor likely generates oligodendrocytes and a subset of the interneurons in the forebrain, and our results suggest that it is the PRP.

Parnavelas (1999) and Price and colleagues $(1991,1993)$ found that the E16 cortex did not contain clones that produced astrocytes and oligodendrocytes, in agreement with the long-term lineage tracing of OLIG1-expressing cells (Lu et al., 2002). However, a recent examination of the same transgenic animal, by another group, has revealed that some astrocytes and radial glia in the spinal cord are generated by OLIG1-expressing precursors (Liu and Rao, 2004). Closer examination of the forebrain may reveal similar findings. In fact, retroviral lineage tracing of the postnatal rat cortex has revealed the existence of precursors with the potential to generate oligodendrocytes and astrocytes in vivo (Levison and Goldman, 1993). It is possible, however, that embryonic and postnatal OLPs represent two 
distinct populations that arise from the MGE and cortical SVZ, respectively. In fact, Ivanova et al. (2003) suggested that these two populations contribute to two temporally distinct waves of cortical myelination. Thus, embryonic OLPs (equivalent to the PRP) may not generate astrocytes, whereas postnatal OLPs may do so actively. Nevertheless, our findings suggest that E14 PRPs can generate astrocytes in vitro, but only when differentiated in the presence of BMP-2 and CNTF signaling. The actions of BMP-2 and CNTF may depend on a complex of the transcription factors Stat 3 and Smad 1 and the coactivator p300/CBP, which have been shown to induce astrocyte differentiation of fetal neural progenitors (Nakashima et al., 1999). A lack of such cooperative signaling may explain previous observations in which BMP signaling failed to promote astroglial differentiation (Wada et al., 2000; Mekki-Dauriac et al., 2002), although this may also be attributable to heterogeneity of OLP populations. Furthermore, our data expand previous observations that OLIG1/2 suppress astrocyte cell fate specification (Zhou and Anderson, 2002) and GFAP expression (Gabay et al., 2003; Fukuda et al., 2004), by our finding that BMP and CNTF signaling that cooperates in the differentiation of astrocytes correlates with the repression of OLIG2 (and perhaps OLIG1) expression. Even if PRPs do not generate astrocytes during embryonic development, their contribution to glial scarring in injury has not been assessed, which leaves the possibility open that PRPs may generate astrocytes in vivo.

Our study has revealed that PRPs have an extensive potential for expansion/self-renewal. FGF-2 has previously been shown to prevent the differentiation of O-2A progenitor cells and promote their self-renewal (Bogler et al., 1990). In contrast, we found that both PDGF and FGF-2 were required for the formation of secondary neurospheres. In addition, we found that PDGF and FGF signaling act through SHH to promote PRP self-renewal. Kessaris et al. (2004) have recently demonstrated that the generation of OLPs by SHH is dependent on a basal level of MAPK activity, which is provided by FGF signaling. Therefore, it is possible that MAPK plays a role in the regulation of $\mathrm{SHH}$ signaling in PRPs and their self-renewal. It is noteworthy that the maximal number of secondary PRPs (26-28 neurospheres), derived from a primary $\mathrm{PRP}$, is approximately equivalent to the number of undifferentiated cells within each PRP clone. Furthermore, undifferentiated cells are mostly eliminated when PRP clones are differentiated into neurons and astrocytes in the presence of BMP-2 plus CNTF, although the neuron numbers are unchanged. Together with the mainly mutually exclusive differentiation of neurons and oligodendrocytes in PRP clones, this leads us to propose a model for the lineage of PRPs (supplemental Fig. 1, available at www.jneurosci.org as supplemental material). PRPs are likely descendents of multipotent NSCs, and this is supported by preliminary findings that show that they can be generated by primary EGFresponsive NSCs and have identical properties to the PRPs from the ventral forebrain studied here (data not shown). The PRP undergoes a series of both symmetric and asymmetric cell divisions, to produce more of itself (self-renewal) and a cell with the potential to differentiate into either a neuron or oligodendrocyte (N/O cell). Our findings suggest that the majority of undifferentiated PRPs (approximately two-thirds of the cells within each clone) will differentiate into astrocytes in the presence of BMP-2 plus CNTF, which results in a reduction in the number of undifferentiated cells without affecting neuron numbers induced by the action of BMP-2 on the N/O cell. Indeed, our contention that astrocyte generation is direct from PRPs and separate from the $\mathrm{N} / \mathrm{O}$ cell is supported by our finding that clones containing both astrocytes and oligodendrocytes are observed when PDGF- generated neurospheres are differentiated in T3, followed by the addition of BMP-2 plus CNTF for the remainder of the differentiation period.

The results of this study suggest that PRPs are a unique population of oligodendrocyte precursors, with both distinct and similar properties to other OLPs described and reviewed previously (Liu and Rao, 2003; Noble et al., 2004; Rowitch, 2004). Therefore, one may ask whether in vitro studies have told us anything useful about the properties of OLPs. It can be argued that the in vitro studies have revealed that these precursors are heterogeneous in their potential to generate neurons and subtypes of astrocytes, and this is dependent on the CNS region and developmental period of isolation. The early development of hindbrain OLPs is unimpaired in OLIG2 null mice ( $\mathrm{Lu}$ et al., 2002), whereas there is a complete absence of OLPs in the spinal cord, suggesting that OLPs in vivo are also a heterogeneous population. Even within the forebrain, we found that there may be heterogeneity in PRPs based on the expression of TOAD-64. Therefore, if forebrain PRPs generate neurons in vivo, it may only be a subpopulation of PRPs that posses this capability. Moreover, it is not certain that PRPs maintain the capacity to generate neurons through to adulthood. Clearly, both in vitro and in vivo studies highlight the need to further define the properties of OLPs throughout development, and studies of spinal cord OLPs may not be predictive of oligodendrocyte biology or pathology in the forebrain and vice versa. The PRP neurosphere system may be a simple and useful way of comparing and contrasting oligodendrocyte precursors in various regions and at various developmental periods, without the requirement of specific cell surface markers, immunopanning, etc. Furthermore, if human PRPs can be similarly generated as neurospheres, this may permit for a straightforward approach to isolating and expanding oligodendrocyte precursors for their transplantation in white matter for the treatment of injury or disease.

\section{References}

Aguirre AA, Chittajallu R, Belachew S, Gallo V (2004) NG2-expressing cells in the subventricular zone are type C-like cells and contribute to interneuron generation in the postnatal hippocampus. J Cell Biol 165:575-589.

Ahlgren SC, Wallace H, Bishop J, Neophytou C, Raff MC (1997) Effects of thyroid hormone on embryonic oligodendrocyte precursor cell development in vivo and in vitro. Mol Cell Neurosci 9:420-432.

Belachew S, Chittajallu R, Aguirre AA, Yuan X, Kirby M, Anderson S, Gallo V (2003) Postnatal NG2 proteoglycan-expressing progenitor cells are intrinsically multipotent and generate functional neurons. J Cell Biol 161:169-186.

Bogler O, Wren D, Barnett SC, Land H, Noble M (1990) Cooperation between two growth factors promotes extended self-renewal and inhibits differentiation of oligodendrocyte-type-2 astrocyte (O-2A) progenitor cells. Proc Natl Acad Sci USA 87:6368-6372.

Cooper MK, Porter JA, Young KE, Beachy PA (1998) Teratogen-mediated inhibition of target tissue response to Shh signaling. Science 280:1603-1607.

Forsberg-Nilsson K, Behar TN, Afrakhte M, Barker JL, McKay RD (1998) Platelet-derived growth factor induces chemotaxis of neuroepithelial stem cells. J Neurosci Res 53:521-530.

Fruttiger M, Karlsson L, Hall AC, Abramsson A, Calver AR, Bostrom H, Willetts K, Bertold CH, Heath JK, Betsholtz C, Richardson WD (1999) Defective oligodendrocyte development and severe hypomyelination in PDGF-A knockout mice. Development 126:457-467.

Fukuda S, Kondo T, Takebayashi H, Taga T (2004) Negative regulatory effect of an oligodendrocytic bHLH factor OLIG2 on the astrocytic differentiation pathway. Cell Death Differ 11:196-202.

Gabay L, Lowell S, Rubin LL, Anderson DJ (2003) Deregulation of dorsoventral patterning by FGF confers trilineage differentiation capacity on CNS stem cells in vitro. Neuron 40:485-499. 
Gomes WA, Mehler MF, Kessler JA (2003) Transgenic overexpression of BMP4 increases astroglial and decreases oligodendroglial lineage commitment. Dev Biol 255:164-177.

Grove EA, Williams BP, Li DQ, Hajihosseini M, Friedrich A, Price J (1993) Multiple restricted lineages in the embryonic rat cerebral cortex. Development 117:553-561.

Gupta SK, Altares M, Benoit R, Riopelle RJ, Dunn RJ, Richardson PM (1992) Preparation and biological properties of native and recombinant ciliary neurotrophic factor. J Neurobiol 23:481-490.

Herrera J, Yang H, Zhang SC, Proschel C, Tresco P, Duncan ID, Luskin M, Mayer-Proschel M (2001) Embryonic-derived glial-restricted precursor cells (GRP cells) can differentiate into astrocytes and oligodendrocytes in vivo. Exp Neurol 171:11-21.

Hughes SM, Lillien LE, Raff MC, Rohrer H, Sendtner M (1988) Ciliary neurotrophic factor induces type- 2 astrocyte differentiation in culture. Nature 335:70-73.

Ivanova A, Nakahira E, Kagawa T, Oba A, Wada T, Takebayashi H, Spassky N, Levine J, Zalc B, Ikenaka K (2003) Evidence for a second wave of oligodendrogenesis in the postnatal cerebral cortex of the mouse. J Neurosci Res 73:581-592.

Kessaris N, Jamen F, Rubin LL, Richardson WD (2004) Cooperation between sonic hedgehog and fibroblast growth factor/MAPK signalling pathways in neocortical precursors. Development 131:1289-1298.

Kondo T, Raff M (2000) Oligodendrocyte precursor cells reprogrammed to become multipotential CNS stem cells. Science 289:1754-1757.

Levison SW, Goldman JE (1993) Both oligodendrocytes and astrocytes develop from progenitors in the subventricular zone of postnatal rat forebrain. Neuron 10:201-212.

Levison SW, Chuang C, Abramson BJ, Goldman JE (1993) The migrational patterns and developmental fates of glial precursors in the rat subventricular zone are temporally regulated. Development 119:611-622.

Liu Y, Rao M (2003) Oligodendrocytes, GRPs and MNOPs. Trends Neurosci $26: 410-412$.

Liu Y, Rao MS (2004) Olig genes are expressed in a heterogeneous population of precursor cells in the developing spinal cord. Glia 45:67-74.

Lu QR, Sun T, Zhu Z, Ma N, Garcia M, Stiles CD, Rowitch DH (2002) Common developmental requirement for Olig function indicates a motor neuron/oligodendrocyte connection. Cell 109:75-86.

Mabie PC, Mehler MF, Marmur R, Papavasiliou A, Song Q, Kessler JA (1997) Bone morphogenetic proteins induce astroglial differentiation of oligodendroglial-astroglial progenitor cells. J Neurosci 17:4112-4120.

Marin O, Rubenstein JL (2001) A long, remarkable journey: tangential migration in the telencephalon. Nat Rev Neurosci 2:780-790.

Marshall CA, Goldman JE (2002) Subpallial dlx2-expressing cells give rise to astrocytes and oligodendrocytes in the cerebral cortex and white matter. J Neurosci 22:9821-9830.

Mekki-Dauriac S, Agius E, Kan P, Cochard P (2002) Bone morphogenetic proteins negatively control oligodendrocyte precursor specification in the chick spinal cord. Development 129:5117-5130.

Minturn JE, Geschwind DH, Fryer HJ, Hockfield S (1995) Early postmitotic neurons transiently express TOAD-64, a neural specific protein. J Comp Neurol 355:369-379.

Mohammadi M, McMahon G, Sun L, Tang C, Hirth P, Yeh BK, Hubbard SR, Schlessinger J (1997) Structures of the tyrosine kinase domain of fibroblast growth factor receptor in complex with inhibitors. Science 276:955-960.

Nakashima K, Yanagisawa M, Arakawa H, Kimura N, Hisatsune T, Kawabata M, Miyazono K, Taga T (1999) Synergistic signaling in fetal brain by STAT3-Smad1 complex bridged by p300. Science 284:479-482.

Nery S, Wichterle H, Fishell G (2001) Sonic hedgehog contributes to oligodendrocyte specification in the mammalian forebrain. Development 128:527-540.

Nishiyama A, Lin XH, Giese N, Heldin CH, Stallcup WB (1996) Colocalization of NG2 proteoglycan and PDGF alpha-receptor on O2A progenitor cells in the developing rat brain. J Neurosci Res 43:299-314.

Noble M, Proschel C, Mayer-Proschel M (2004) Getting a GR(i)P on oligodendrocyte development. Dev Biol 265:33-52.

Ono K, Bansal R, Payne J, Rutishauser U, Miller RH (1995) Early development and dispersal of oligodendrocyte precursors in the embryonic chick spinal cord. Development 121:1743-1754.
Orentas DM, Hayes JE, Dyer KL, Miller RH (1999) Sonic hedgehog signaling is required during the appearance of spinal cord oligodendrocyte precursors. Development 126:2419-2429.

Parnavelas JG (1999) Glial cell lineages in the rat cerebral cortex. Exp Neurol 156:418-429.

Price J, Thurlow L (1988) Cell lineage in the rat cerebral cortex: a study using retroviral-mediated gene transfer. Development 104:473-482.

Pringle NP, Richardson WD (1993) A singularity of PDGF alpha-receptor expression in the dorsoventral axis of the neural tube may define the origin of the oligodendrocyte lineage. Development 117:525-533.

Pringle NP, Yu WP, Guthrie S, Roelink H, Lumsden A, Peterson AC, Richardson WD (1996) Determination of neuroepithelial cell fate: induction of the oligodendrocyte lineage by ventral midline cells and sonic hedgehog. Dev Biol 177:30-42.

Rao MS, Mayer-Proschel M (1997) Glial-restricted precursors are derived from multipotent neuroepithelial stem cells. Dev Biol 188:48-63.

Rao MS, Noble M, Mayer-Proschel M (1998) A tripotential glial precursor cell is present in the developing spinal cord. Proc Natl Acad Sci USA 95:3996-4001.

Represa A, Shimazaki T, Simmonds M, Weiss S (2001) EGF-responsive neural stem cells are a transient population in the developing mouse spinal cord. Eur J Neurosci 14:452-462.

Reuss B, von Bohlen und Halbach O (2003) Fibroblast growth factors and their receptors in the central nervous system. Cell Tissue Res 313:139-157.

Reynolds BA, Weiss S (1996) Clonal and population analyses demonstrate that an EGF-responsive mammalian embryonic CNS precursor is a stem cell. Dev Biol 175:1-13.

Reynolds BA, Tetzlaff W, Weiss S (1992) A multipotent EGF-responsive striatal embryonic progenitor cell produces neurons and astrocytes. J Neurosci 12:4565-4574.

Rowitch DH (2004) Glial specification in the vertebrate neural tube. Nat Rev Neurosci 5:409-419.

Shimazaki T, Shingo T, Weiss S (2001) The ciliary neurotrophic factor/leukemia inhibitory factor/gp130 receptor complex operates in the maintenance of mammalian forebrain neural stem cells. J Neurosci 21:7642-7653.

Taipale J, Chen JK, Cooper MK, Wang B, Mann RK, Milenkovic L, Scott MP, Beachy PA (2000) Effects of oncogenic mutations in Smoothened and Patched can be reversed by cyclopamine. Nature 406:1005-1009.

Tekki-Kessaris N, Woodruff R, Hall AC, Gaffield W, Kimura S, Stiles CD, Rowitch DH, Richardson WD (2001) Hedgehog-dependent oligodendrocyte lineage specification in the telencephalon. Development 128:2545-2554.

Temple S, Raff MC (1985) Differentiation of a bipotential glial progenitor cell in a single cell microculture. Nature 313:223-225.

Tropepe V, Sibilia M, Ciruna BG, Rossant J, Wagner EF, van der Kooy D (1999) Distinct neural stem cells proliferate in response to EGF and FGF in the developing mouse telencephalon. Dev Biol 208:166-188.

Wada T, Kagawa T, Ivanova A, Zalc B, Shirasaki R, Murakami F, Iemura S, Ueno N, Ikenaka K (2000) Dorsal spinal cord inhibits oligodendrocyte development. Dev Biol 227:42-55.

Warf BC, Fok-Seang J, Miller RH (1991) Evidence for the ventral origin of oligodendrocyte precursors in the rat spinal cord. J Neurosci 11:2477-2488.

Wichterle H, Turnbull DH, Nery S, Fishell G, Alvarez-Buylla A (2001) In utero fate mapping reveals distinct migratory pathways and fates of neurons born in the mammalian basal forebrain. Development 128:3759-3771.

Williams BP, Read J, Price J (1991) The generation of neurons and oligodendrocytes from a common precursor cell. Neuron 7:685-693.

Yung SY, Gokhan S, Jurcsak J, Molero AE, Abrajano JJ, Mehler MF (2002) Differential modulation of BMP signaling promotes the elaboration of cerebral cortical GABAergic neurons or oligodendrocytes from a common sonic hedgehog-responsive ventral forebrain progenitor species. Proc Natl Acad Sci USA 99:16273-16278.

Zhou Q, Anderson DJ (2002) The bHLH transcription factors OLIG2 and OLIG1 couple neuronal and glial subtype specification. Cell 109:61-73. 